\title{
Réaction à un composé salivaire et tolérance à la coupe de génotypes de Dactylis glomerata $L$ issus de populations ayant une expérience contrastée du pâturage
}

\author{
M Fily ${ }^{1,}$ C Tosca ${ }^{1}, \mathrm{G}$ Balent 2 \\ 1 Université Toulouse III, laboratoire d'écologie végétale, 39, allée Jules Guesde, 31062 Toulouse cedex; \\ 2 INRA, centre de recherche de Toulouse, URSAD BP 27, 31326 Castanet-Tolosan cedex, France
}

(Reçu le 9 avril 1990; accepté le 5 février 1991)

\begin{abstract}
Résumé - Une étude comparative est réalisée sur 8 génotypes de dactyle (Dactylis glomerata L) récoltés dans 2 parcelles des Pyrénées qui ne diffèrent que par l'intensité du pâturage qui s'y exerce depuis 30 ans. Nous montrons, à l'aide de mesures de biomasses effectuées sur des plantes cultivées en pots à partir d'une talle unique :

- que certains génotypes tolèrent mieux que d'autres la coupe, simulant le pâturage;

- que la thiamine, mimant la salive des bovins, n'a pas d'effet constant sur la repousse des plantes;

- qu'il y a une cohérence entre la capacité de repousse des génotypes et l'expérience que les populations ont du pâturage depuis 30 ans.

Des notations du stade phénologique (structure morphologique) lors de chaque coupe permettent d'expliquer le cas particulier d'un génotype présentant une photosynthèse nette particulièrement forte en fin d'expérience (15 semaines après l'installation) : les plantes correspondant à ce génotype avaient en effet épié avant la première coupe. On en conclut que le stade phénologique des plantes est très important à considérer pour l'examen de leur capacité de repousse et qu'il doit être pris en compte avant de conclure à des réactions photosynthétiques spécifiques à la défoliation.
\end{abstract}

biomasse / défoliation / thiamine / herbivore / repousse / photosynthèse

Summary - Examination of potential effect of a salivary chemical and defoliation tolerance of Dactylis glomerata $L$ genotypes with diverse histories of grazing management. Eight genotypes of Dactylis glomerata $L$ plants were obtained from 2 more or less heavily grazed grasslands (grazed for $30 \mathrm{yr}$ ). Yield of individual plants produced from a single tiller were compared (table l) and it was observed that :

- some genotypes are more tolerant to clipping (simulated grazing) than others (figs 1, 2);

- thiamine, simulating cattle saliva has no constant effect on regrowth (fig 1);

- clipping tolerance of genotypes was consistent with the grazing experience of population over a 30-yr period (fig 1).

Phenologic records for each cutting provided an explanation for the case of a genotype ("D», fig 4) which exhibited high net photosynthesis at the end of the experiment (15 wk after transplantation). These plants produce fertile tillers before the first cut. We conclude that the phenologic stage of plants is very important to consider in the examination of regrowth capacity and must be taken into consideration before putting forward a conclusion relating to specific photosynthetic response to defoliation.

biomass / defoliation / thiamine / herbivore / regrowth / photosynthesis

\section{INTRODUCTION}

Les populations végétales fortement pâturées se caractérisent par des individus plus petits et plus prostrés que ceux provenant de populations non pâturées. Dans de nombreux cas, il a été montré que ces différences morphologiques sont déter- minées génétiquement et qu'elles se maintiennent durant plusieurs années en environnement commun (Peterson, 1962; Detling et al, 1986). Cette micro-différenciation sous l'effet du pâturage survient en peu de temps et sur de faibles distances (15 ans et quelques dizaines de $\mathrm{m}$ McNaughton, 1979). 
Des études morphométriques existent sur le dactyle (Stapledon, 1928; Kemp, 1937; Rebischung, 1953; Van Dijk, 1955; Charles, 1961; Fily, 1990). Mais la variabilité de la repousse elle-même est étudiée depuis moins longtemps (Madembo, 1986), que ce soit au niveau du résultat (= la production de biomasse) ou au niveau des mécanismes (= la photosynthèse par exemple). Or, la capacité de repousse est particulièrement intéressante à connaître, tant du point de vue de l'évaluation de la ressource fourragère que de celui du végétal lui-même dont le statut compétitif au sein de la communauté dépend fortement de la taille.

Le premier but de cette étude consistera donc à comparer la tolérance à la coupe de différents génotypes de dactyle.

Pour évaluer cette tolérance, nous utiliserons la méthode classique de la coupe tout en sachant que cette défoliation mécanique ne mime qu'imparfaitement (Archer et Tieszen, 1980) le pâturage qui est le seul mode d'exploitation que connaissent les plantes in situ dans les parcelles sélectionnées.

Pour une plante, le dépôt de salive par les animaux lors de la prise alimentaire figure parmi les principales différences entre la coupe et le pâturage. Le deuxième but de cette étude consistera à évaluer la réaction des plantes à un dépôt d'un composé salivaire, la thiamine. Nous y voyons 2 intérêts : premièrement, la stimulation de la repousse par la salive pourrait atteindre $40 \%$ de la biomasse de plantes isolées (Reardon et al, 1972, 1974; McNaughton, 1985) et, bien que la transposition au niveau de la productivité du peuplement soit délicate, on imagine la sousestimation du rendement qui peut correspondre à cet effet; deuxièmement, sur un aspect plus théorique des relations plantes-herbivores, McNaughton (1985) signale le caractère provoquant d'un mécanisme où des molécules rares, mais ayant une forte activité biologique, sont transférées des herbivores vers les plantes, à contre-courant des flux principaux de masses et d'énergie ce qui contribue à faire apparaître les plantes comme partenaires actifs du couple "herbe-animal".

De plus, 2 observations de terrain nous ont conduits à réaliser cette étude dans une vallée des Pyrénées centrales, par ailleurs bien connue, tant du point de vue de la flore que de I'histoire pastorale (Balent, 1986). Tout d'abord, nous avons remarqué la bonne tolérance de certaines prairies à une très forte pression de pâturage hivernal. Ensuite, le dactyle, observé dans des parcelles très différentes par leurs histoires culturales, est très variable morphologiquement.

Nous pouvons résumer les objectifs de cette étude en formulant les hypothèses suivantes :

- le comportement (biomasse, photosynthèse) des pieds de dactyle vis-à-vis de la coupe et de la thiamine est, pour partie, déterminé génétiquement;

- le pâturage favorise le type le plus tolérant à la défoliation, autrement dit, il y a une cohérence entre l'expérience préalable des populations de dactyle vis-à-vis du pâturage et le comportement des individus qui les composent aujourd'hui. $\mathrm{Ce}$ comportement sera étudié ici par le biais de la biomasse cumulée et de la cinétique de la photosynthèse lors d'une repousse.

\section{MATÉRIEL ET MÉTHODES}

\section{Les 2 populations étudiées}

Ces populations se sont développées spontanément (absence de semis - Balent et Duru, 1984) sur 2 anciens champs localisés aux abords immédiats du village de Oô (Haute-Garonne, France). Les 2 stations, non perturbées depuis 30 ans, sont très semblables par: le substrat (rankers établis sur alluvions glaciaires fortement remaniées par la construction de terrasses; $\mathrm{pH}$ légèrement inférieur à 6 - Nègre, 1972), l'exposition (versant sud), l'altitude $(1000 \mathrm{~m})$ et bien entendu le climat qui se rattache au climat océanique d'abri (Izard, 1985). Les 2 stations sont distantes de $1500 \mathrm{~m}$ et séparées par une mosaïque de parcelles et quelques haies. Elles se différencient essentiellement par le critère de la pression de pâturage passée et présente : nous appellerons Ip30 la population légèrement pâturée depuis 30 ans et FP30 la population fortement pâturée depuis la même date.

Le site d'étude a été présenté de manière complète par ailleurs (Balent et Duru, 1984).

\section{Dispositif expérimental : facteurs et modalités}

Notre expérience comprend 4 facteurs :

- le facteur "population d'origine" avec 2 modalités comme nous venons de le voir. Ce facteur est synonyme d'un facteur «histoire des stations vis-à-vis du pâturage", Ip30 a été peu pâturée, FP30 l'a été fortement;

- le facteur "génotype": le nombre de modalités (1 modalité $=1$ génotype $=1$ individu génétique) par station est réduit à 4 en raison de contraintes logistiques. 
- le facteur "coupe» connaît 3 modalités : outre le témoin "0 coupe», le pâturage lâche est mimé par une réduction de la hauteur des plants à $8 \mathrm{~cm}$ tous les $21 \mathrm{j}$; la coupe la plus sévère (réduction à $5 \mathrm{~cm}$ tous les $15 \mathrm{j}$ ) n'est pas toutefois aussi intense que celle que subissent les plantes en condition de pâturage in situ (Després et Soulas, 1981). Ces modalités de coupe ont été choisies aussi d'après la bibliographie (McNaughton, 1979; Detling et al, 1979; Detling et al, 1980);

- le facteur «thiamine»: la quantité de thiamine apportée est très faible : $0,2 \mathrm{ml}$ d'une solution à $10 \mathrm{ppb}$ (partie pour billion). Elle correspond à la quantité déposée par un animal selon Reardon et al (1974) et McNaughton (1985). Le témoin est obtenu par l'application de la même quantité d'eau distillée.

Le facteur "génotype" est hiérarchisé sous le facteur «population» (Rognli, 1987) et le modèle "("génotype" < «population») $x$ coupe $x$ thiamine» été soumis à une analyse de variance.

Un tel dispositif factoriel présente l'avantage de permettre de tester l'interaction entre les facteurs; celle entre le facteur "génotype" et les facteurs "coupe" et "apport de thiamine» nous intéresse en premier lieu.

Les mesures de biomasse ont été faites sur toutes les plantes. La cinétique de la photosynthèse n'a été déterminée que sur 2 génotypes coupés sévèrement pour chacune des populations, soit 4 génotypes.

\section{Installation et conduite de la culture}

Quatre touffes de dactyle ont été prélevées le 12 mars 1987 à 4 endroits différents de chacune des 2 parcelles. Nous avons choisi des touffes de taille suffisante pour pouvoir y prélever 6 talles de vigueurs comparables. Le dispositif expérimental impose de disposer de ce nombre d'individus génétiques identiques ( 3 modalités de coupe $\times 2$ modalités de thiamine $=6$ talles). Afin d'augmenter encore la ressemblance des talles au début de l'expérience nous avons retaillé les feuilles et les racines de chaque talle avant de les installer, isolées, dans un pot contenant un mélange soigneusement homogénéisé de $50 \%$ de terre végétale et $50 \%$ de terreau. Les 48 pots ont été disposés au hasard et suffisamment loin les uns des autres pour qu'il n'y ait pas de compétition entre les plantes. Celles-ci ont été arrosées très régulièrement en fonction de la demande climatique. Dans ces conditions, nous n'avons pas observé de perte de matériel durant les 2 mois de l'expérience, même sur les plantes non coupées. L'expérimentation a été menée au jardin des plantes de l'université de Toulouse.

La première coupe est intervenue le 18 mai, soit 2 mois après l'installation, et les suivantes se sont déroulées sur 7 semaines ( 3 coupes lâches et 4 coupes sévères auxquelles correspondent 2 et 3 phases de repousse). Dans les heures qui ont suivi la dernière coupe à hauteur précise, toutes les plantes ont été recoupées (coupées pour le témoin) au collet. La cinétique de la photosynthèse a été mesurée lors de la troisième repousse consécutive à une coupe sévère.

\section{Les variables enregistrées}

Nous avons enregistré 2 variables pour chaque plante :

\section{La biomasse cumulée}

Le choix de cette variable se justifie de 2 manières. C'est d'abord le paramètre qui intéresse le plus l'animal et, au-delà, l'éleveur ou l'agronome. C'est ensuite, du point de vue des plantes (phytocentric overview, Dirzo, 1984), un des meilleurs indicateurs de leur adaptation à l'habitat pâturé puisque l'accumulation de matériel témoigne des capacités compétitives qui sont particulièrement importantes dans les prairies (Donald, 1963).

C'est pourquoi, privilégiant le «point de vue des plantes" plutôt que celui de l'éleveur ou de l'agronome plus habitués à raisonner en terme de biomasse récoltable, nous avons enregistré la matière sèche aérienne cumulée durant la totalité de l'expérience (production des coupes successives et de la coupe finale au collet). La matière sèche est pesée après un séjour à l'étuve de $72 \mathrm{~h}$ à $80^{\circ} \mathrm{C}$.

\section{La photosynthèse lors d'une repousse}

Au niveau de la plante entière, la repousse plus vigoureuse d'un génotype pourrait s'expliquer, notamment, par une récupération plus rapide de l'activité photosynthétique. Nous avons donc déterminé la cinétique de cette activité photosynthétique pour 8 individus.

Le système de mesure comprend une chambre d'assimilation qui enferme les parties aériennes et dans laquelle circule en continu de l'air à débit constant. Les variations de teneur en $\mathrm{CO}_{2}$ de l'air circulant sont enregistrées par un analyseur de gaz à infrarouge (modèle ANIR-12 de Schlumberger). Nous nous sommes inspirés de Williams et Kemp (1978) pour la construction de chambres d'assimilation qui assurent une séparation étanche entre les parties aériennes et les parties souterraines des plantes. Le joint au niveau du collet est renforcé par de la pâte à modeler, ce qui permet d'éliminer efficacement la contamination de la chambre supérieure par le gaz de la partie inférieure et ce, en dépit du port en touffe du dactyle. Lors des mesures effectuées en phytotron l'éclairement incident était de 20000 lux (420 W. $\mathrm{m}^{-2}$ ) et la température de $18^{\circ} \mathrm{C}$. Enfin les plantes étaient uniformément arrosées quelques $h$ avant la séance de mesure.

Chaque point expérimental est obtenu par différence entre 2 enregistrements : le premier est effectué à l'obscurité et permet de calculer la respiration obscure; puis une seconde mesure est faite à la lumière, la variation de teneur en $\mathrm{CO}_{2}$ de l'air est alors le bilan de la photosynthèse, de la respiration obscure et de la photorespiration. Par différence avec la première mesure on obtient la photosynthèse apparente 
$(P a)$, synonyme de photosynthèse nette $(P n)$. Nous avons suivi Sestak et al (1971) pour le calcul de l'activité photosynthétique « $\mathrm{F} »$, exprimée en $\mathrm{g}$ de $\mathrm{CO}_{2}$ par individu et par s.

Les cinétiques sont suivies pendant 11 j et construites à partir de 7 points expérimentaux. Le premier de ces points est obtenu moins de $6 \mathrm{~h}$ avant la défoliation, le second est noté dans l'heure qui suit la coupe. Lors de la phase de mise au point de l'expérimentation, nous avons répété les mesures sur un échantillon à quelques dizaines de min d'intervalle. Elles se sont révélées très reproductibles; en conséquence, nous n'avons réalisé qu'une mesure pour déterminer chaque point de la cinétique.

Outre ces 2 variables principales, nous avons noté lors de chaque coupe la vigueur (échelle de 0 à 5) de chaque plante et le nombre de talles épiées.

\section{RÉSULTATS}

\section{Biomasse}

Le tableau I regroupe les mesures effectuées sur les 48 plantes ( $g$ de MS par plante). On notera des écarts relativement forts entre ces valeurs absolues.

Tableau I. Biomasse cumulée et biomasse résiduelle (en italique) à l'issue de la dernière coupe pour chaque plante $(\mathrm{g})$. Apport de thiamine : $(+)$; témoin sans thiamine : $(-)$.

\begin{tabular}{|c|c|c|c|c|c|c|c|}
\hline \multirow[t]{3}{*}{$\begin{array}{l}\text { Popu- } \\
\text { lation }\end{array}$} & \multirow[t]{3}{*}{$\begin{array}{l}\text { Géno } \\
\text { type }\end{array}$} & \multicolumn{2}{|c|}{$\begin{array}{r}\text { Coupe } \\
\text { absente }\end{array}$} & \multicolumn{2}{|c|}{$\begin{array}{l}\text { Coupe } \\
\text { lâche }\end{array}$} & \multicolumn{2}{|c|}{$\begin{array}{l}\text { Coupe } \\
\text { sévère }\end{array}$} \\
\hline & & \multicolumn{6}{|c|}{ thiamine } \\
\hline & & + & - & + & - & + & - \\
\hline Ip $30 \mathrm{~A}$ & & 4,34 & 4,23 & 2,38 & 2,82 & 1,97 & 1,62 \\
\hline Ip $30 \mathrm{~B}$ & & 3,71 & 4,48 & 2,25 & 1,97 & 1,55 & 2,41 \\
\hline IP $30 \mathrm{C}$ & & 2,29 & 2,21 & $\begin{array}{l}1,88 \\
0,97\end{array}$ & $\begin{array}{l}3,36 \\
1,63\end{array}$ & $\begin{array}{l}1,23 \\
0,58\end{array}$ & $\begin{array}{l}1,19 \\
0,51\end{array}$ \\
\hline IP $30 \quad D$ & & 5,09 & 5,72 & $\begin{array}{l}2,28 \\
1,21\end{array}$ & $\begin{array}{l}2,51 \\
1,67\end{array}$ & $\begin{array}{l}1,27 \\
0,72\end{array}$ & $\begin{array}{l}1,17 \\
0,41\end{array}$ \\
\hline $\mathrm{FP} 30 \mathrm{E}$ & & 3,43 & 3,64 & $\begin{array}{l}2,7 \\
0,91\end{array}$ & $\begin{array}{l}3,30 \\
1,51\end{array}$ & $\begin{array}{l}1,16 \\
0,60\end{array}$ & $\begin{array}{l}1,46 \\
0,53\end{array}$ \\
\hline $\mathrm{FP} 30 \mathrm{~F}$ & & 2,59 & 1,93 & $\begin{array}{l}3,11 \\
1,94\end{array}$ & $\begin{array}{l}2,69 \\
1,94\end{array}$ & $\begin{array}{l}1,71 \\
0,81\end{array}$ & $\begin{array}{l}1,37 \\
0,62\end{array}$ \\
\hline FP $30 \mathrm{G}$ & & 3,76 & 3,08 & $\begin{array}{l}2,93 \\
1,08\end{array}$ & $\begin{array}{r}4,10 \\
1,72\end{array}$ & $\begin{array}{l}2,94 \\
0,67\end{array}$ & $\begin{array}{l}3,98 \\
1,35\end{array}$ \\
\hline $\mathrm{FP} 30 \mathrm{H}$ & & 7,12 & 10,42 & $\begin{array}{l}5,11 \\
3,05\end{array}$ & $\begin{array}{l}7,40 \\
4,44\end{array}$ & $\begin{array}{l}5,47 \\
1,02\end{array}$ & $\begin{array}{l}5,52 \\
2,03\end{array}$ \\
\hline
\end{tabular}

La figure 1 représente de manière synthétique l'effet des traitements sur les différents génotypes. La base 100 est obtenue pour chaque génotype par le témoin non coupé.

On notera :

- que les réactions sont très variables d'un génotype à l'autre. Avec une coupe sévère, le clone $D$ voit sa biomasse réduite à $20 \%$ de la valeur du témoin non coupé alors que le clone $F$, avec une coupe lâche, voit sa biomasse augmenter jusqu'à atteindre 120 et $139 \%$ du témoin. Mais la valeur relative la plus forte est observée pour le clone $\mathrm{C}$ qui provient de la parcelle la moins pâturée;

- que, en général, la coupe lâche diminue moins la production que la coupe sévère;

- que la production moyenne de la population historiquement peu pâturée est plus déprimée par les 2 régimes de coupe que celle de la population connaissant un fort pâturage depuis 30 ans. En effet, les individus de la première population ne «récupèrent» que 38,7 et $60 \%$ de biomasse du lot témoin, respectivement pour la coupe sévère et lâche, alors que pour la seconde population les valeurs correspondantes sont égales à 65,6 et $87 \%$;

- que la thiamine n'a pas d'effet constant (des stimulations mais surtout 3 fois sur 16 un fort effet dépressif).

La figure 2 permet de mieux suivre la réponse à la coupe de chaque génotype ainsi que leur production totale, les 2 échantillons, thiamine (+) et thiamine (-), sont cumulés.

$-H$, bien que déprimé par la coupe, reste le plus productif,

- les génotypes collectés en Ip30 ont une réponse à la coupe beaucoup plus homogène que ceux collectés en FP30,

- les effets apparemment positifs ( $F$ et $G$ ) correspondent aux génotypes dont les individus non coupés sont les moins productifs (voir aussi tableau I).

\section{Analyse de variance}

\section{Transformation de données}

Nous avons procédé à une transformation logarithmique des biomasses cumulées (incluant la biomasse résiduelle, du collet à 5 ou $8 \mathrm{~cm}$ de hauteur) afin d'améliorer l'ajustement de la distribution des résidus à une loi normale. Cette transformation est surtout justifiée par le fait que nous 

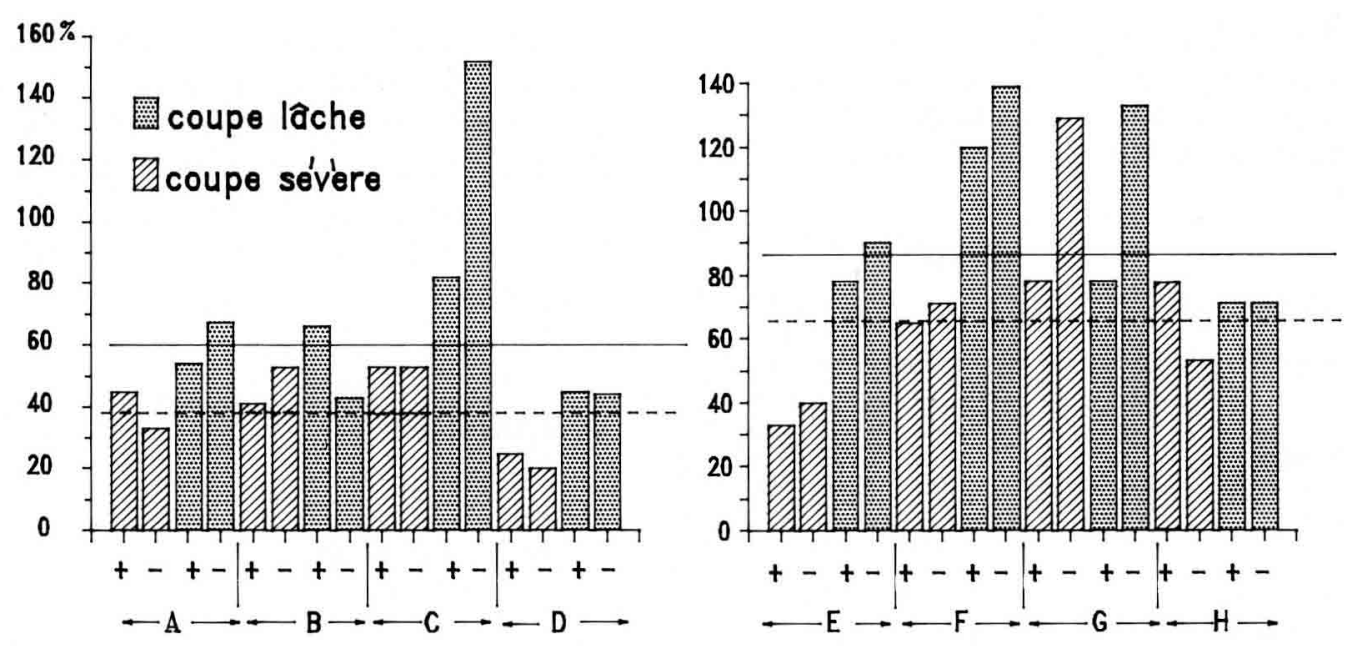

Stations d'origine:

$\operatorname{lp} 30$

FP 30

Fig 1. Pourcentage de biomasse cumulée par rapport au témoin non coupé (base 100) et moyennes pour chaque régime de coupe : coupe lâche : - (toutes les 3 semaines à $8 \mathrm{~cm}$ du sol); coupe sévère : - - (toutes les 2 semaines à $5 \mathrm{~cm}$ du sol); génotypes : A, B, C, D, E, F, G, H. Apport de thiamine (+); témoin sans thiamine: (-).

\section{biomasse $\mathrm{g}$ \\ (somme des 2 échantillons \\ thiamine $(+)$ et $(-))$}

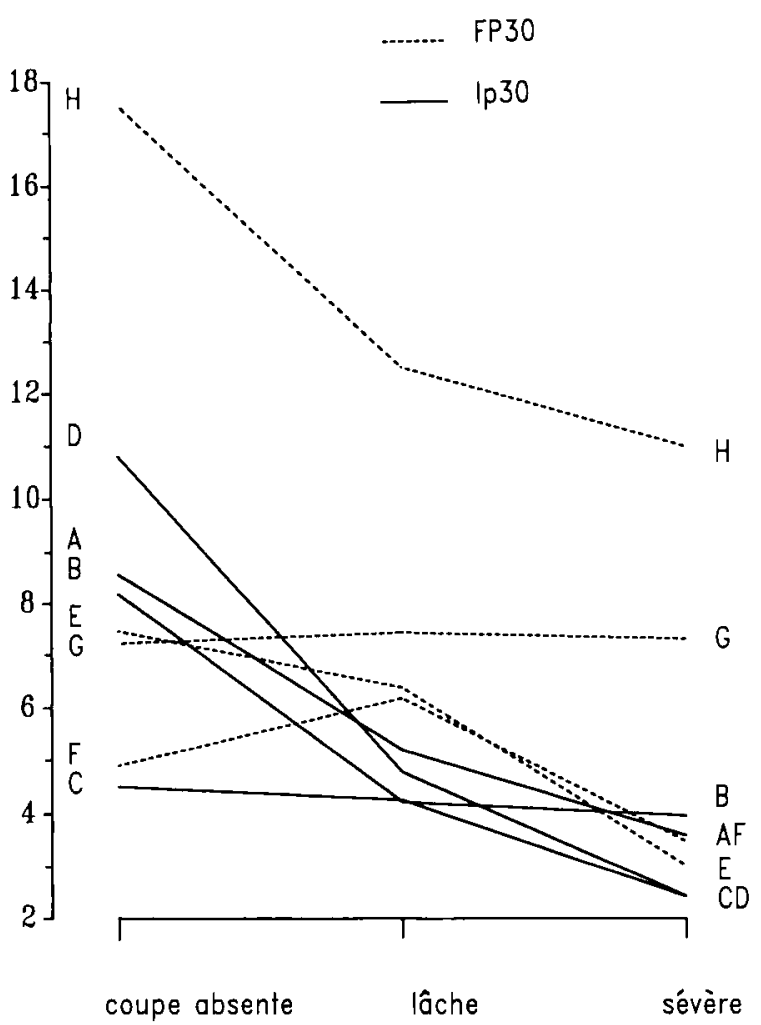

Fig 2. Biomasse cumulée (en ordonnée) pour chaque génotype et pour chaque régime de coupe (en abscisse). Les valeurs des 2 échantillons, thiamine (+) et thiamine (-), sont cumulées. comparons 48 plantes isolées sur le critère de leur croissance. Le mécanisme de croissance, dans ces conditions d'absence de compétition, se rapproche d'un modèle exponentiel. La transformation logarithmique se trouve donc justifiée à la fois par des arguments statistiques et biologiques (Dunn, 1981). Cette transformation est d'ailleurs très fréquente dans ce type d'études (Madembo, 1986; Aarssen et Turkington, 1987; Jaramillo et Detling, 1988).

\section{(Génotype < Population) x coupe $\times$ thiamine (tableau II)}

Les éléments les plus intéressants sont : d'une part, les interactions hautement significatives (au seuil $\alpha=0,01$ ) entre le facteur coupe et les facteurs "génotype" et "population", et, d'autre part, l'absence d'effet significatif de la thiamine $(\alpha=0,09$, tendance à un effet négatif visible dans le tableau I).

Sur les seules plantes non coupées (résultats non figurés), le facteur génotype reste hautement significatif. Rappelons que les 3 témoins les moins productifs sont ceux qui tolèrent le mieux la coupe.

\section{Photosynthèse lors d'une repousse}

La figure 3 représente les cinétiques observées pour chaque génotype. 
Tableau II. Effets de la coupe de la thiamine et de la population d'origine sur la repousse de génotypes de dactyle (les données sont des mesures de matière sèche cumulée après avoir subi une transformation logarithmique).

\section{Source de variation ddl $F$ Probabilité}

$\begin{array}{lrrl}\text { Population } & 1 & 42,97 & 0,0001 \\ \text { (génotype < pop) } & 6 & 30,24 & 0,0001 \\ \text { Coupe } & 2 & 69,86 & 0,0001 \\ \text { Thiamine } & 1 & 3,32 & 0,0934 \\ \text { Population x coupe } & 2 & 9,47 & 0,0034 \\ \text { Population x thiamine } & 1 & 0,00 & 0,96 \\ \text { (Génotype < pop) x coupe } & 12 & 4,71 & 0,0059 \\ \text { (Génotype < pop) x thiamine } & 6 & 1,40 & 0,2916 \\ \text { Coupe x thiamine } & 2 & 1,03 & 0,3881 \\ \text { Population x thiamine x coupe } & 2 & 0,14 & 0,8711 \\ \text { Résiduelle } & 12 & & \end{array}$

Ces cinétiques sont particulièrement variées et il est difficile de reconnaître des modes de réaction nets. Nous remarquerons que dans 6 cas sur 8 l'activité décroît de manière nette du fait de la défoliation. Dans les 2 autres cas la Pn n'a pas variée $3 \mathrm{~h}$ après la coupe. Pour l'individu Dcette absence de réaction s'explique par le fait que la coupe a enlevé du matériel peu actif-des tiges essentiellement- (nous n'avons pas d'explication concernant le comportement de $F_{+}$). La tendance générale est ensuite au recouvrement de l'activité initiale, voire à son dépassement avant la fin du suivi qui dure $11 \mathrm{j}$. On notera une plus grande similarité des courbes correspondant à un même génotype, qu'il ait reçu ou non de la thiamine.
Cette ressemblance est plus nette encore (analyse de variance : effet génotype significatif à $\alpha=0,001$ ) sur la base des activités photosynthétiques ramenées à l'unité de biomasse au $9^{e} \mathrm{j}$ de la repousse (fig 4 : les valeurs de biomasse utilisées sont celles obtenues lors de la coupe effectuée le $11^{e} \mathrm{j}$ ).

\section{DISCUSSION \\ Biomasse cumulée}

\section{Effet de la coupe}

II n'est pas surprenant de noter un effet hautement significatif de la coupe étant donné le contraste entre les modalités qui étaient appliquées pour l'étude de ce facteur. II est plus instructif de se pencher sur l'effet de chaque modalité de ce facteur «coupe».

La coupe lâche diminue moins la production des plantes que la coupe sévère et ce de manière constante quel que soit le génotype. Nous ne pouvons toutefois pas généraliser en affirmant que «moins un individu est coupé plus il produit» car les génotypes $F$ (et de manière moins nette les génotypes $\mathrm{G}$ et $\mathrm{C}$ ) ont une production finale supérieure aux témoins non coupés. Il y a dans ces cas une croissance non seulement compensatrice mais surcompensatrice (McNaughton, 1983, 1986).

Au-delà des 3 cas particuliers de surcompensation apparente, l'interaction hautement significative entre la coupe et les génotypes résulte du fait que les génotypes les plus résistants à une coupe lâche sont aussi ceux qui tolèrent le mieux

Tableau III. Travaux traitant de l'effet de la salive des herbivores sur la repousse des plantes consommées $(P n=$ photosynthèse nette, $R r=$ respiration raçinaire, ${ }^{14} \mathrm{C}=$ mouvement de photosynthétats marqués par l'isotope ${ }^{14} \mathrm{C}$. (1) McNaughton (1985); (2) Reardon et al (1972); (3) Reardon et al (1974); (4) Johnston et Bailey in: Detling et al (1980); (5) Detling et al (1980); (6) Detling et al (1981).

\begin{tabular}{|c|c|c|c|c|}
\hline Élément apporté & Espèce végétale & Fréq coupes & Réaction & luteurs \\
\hline Thiamine & Sporobolus iocladus/S pyramidalis & $5 \mathrm{~cm} / 1$ semaine & biomasse, sucres stimulés & (1) \\
\hline Salive bovin & Bouteloua curtipendula & - & biomasse stimulée & (2) \\
\hline Salive bovin & Bouteloua curtipendula & $7-15 \mathrm{~cm} / 3-6 \mathrm{sem}$ & sans & (3) \\
\hline Thiamine & $\begin{array}{l}\text { idem } \\
\text { idem }\end{array}$ & $\begin{array}{l}15 \mathrm{~cm} / 6 \text { semaines } \\
7 \mathrm{~cm} / 3 \text { semaines }\end{array}$ & $\begin{array}{l}\text { biomasse stimulée } \\
\text { sans }\end{array}$ & $\begin{array}{l}\text { (3) } \\
\text { (3) }\end{array}$ \\
\hline Salive bovin & 2 Festuca & & sans & (4) \\
\hline Salive bison & Bouteloua gracilis & & sans $\left(\mathrm{Pn}, \mathrm{Rr},{ }^{14} \mathrm{C}\right)$ & (5) \\
\hline Salive bison & Avena sativa & bioessai : $1 \mathrm{j}$ & croissance stimulée & (6) \\
\hline Sels (NaHCO3) & Avena sativa & bioessai : $1 \mathrm{j}$ & croissance stimulée & (6) \\
\hline
\end{tabular}



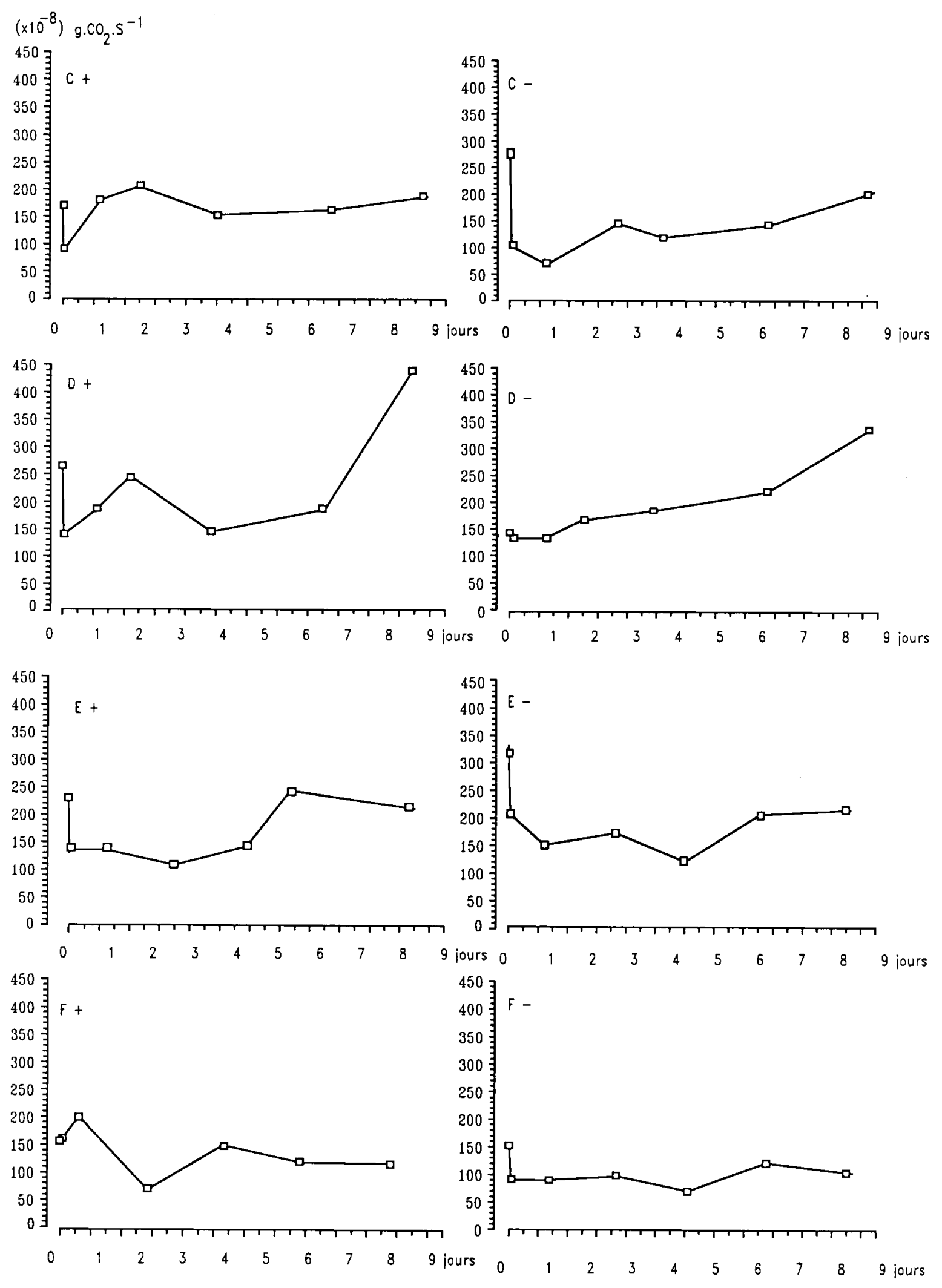

Fig 3. Cinétique de la photosynthèse apparente $\left(g\left(\mathrm{CO}_{2}\right) \cdot \mathrm{s}^{-1}\right)$ de 4 génotypes, lors de la troisième repousse. Génotypes : $\mathrm{C}, \mathrm{D}, \mathrm{E}, \mathrm{F}$; apport de thiamine : $(+)$; témoin sans thiamine : $(-)$. 


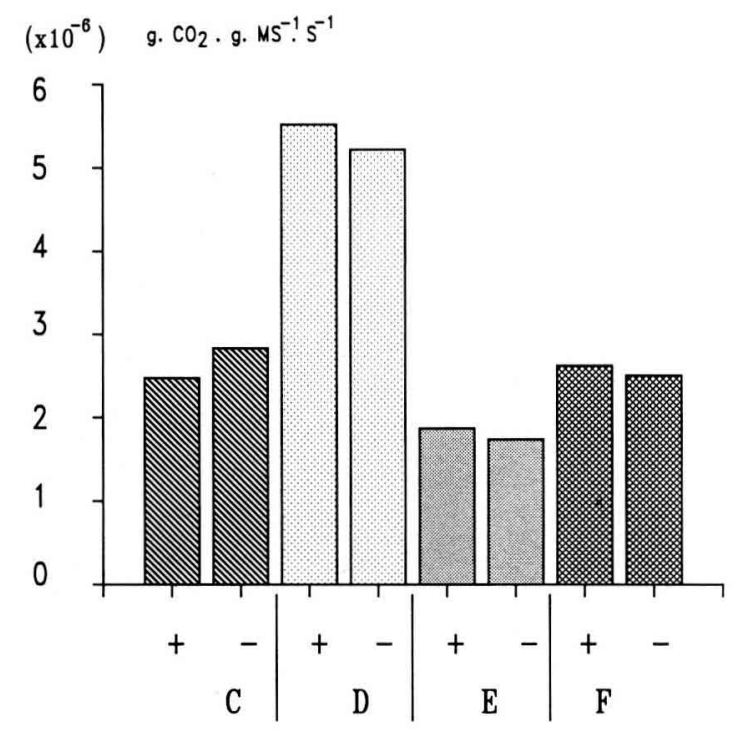

Population Ip $30 \quad$ Population FP 30

Fig 4. Photosynthèse apparente par unité de poids sec (g (CO2) $\mathrm{g}^{-1}(\mathrm{MS}) \cdot \mathrm{s}^{-1}$ ) pour 4 génotypes, le $9^{e} \mathrm{j}$ après la $3^{\mathrm{e}}$ coupe. Symboles identiques à figure 1.

une coupe sévère. Ce résultat plaide donc pour une forte détermination génétique de la tolérance à la coupe sans préjuger des mécanismes en jeu et que nous envisagerons plus loin. De telles différences intergénotypiques sur la base de la biomasse avaient déjà été mises en évidence pour le dactyle par Tinker (1930).

Par ailleurs, les différences significatives observées entre les plantes non coupées ( 8 génotypes) pourraient résulter d'un coût associé à la capacité de repousse; en effet les 3 génotypes dont la production paraît stimulée par la coupe sont ceux dont les productions sont les plus faibles en l'absence de coupe. Nos résultats concordent avec ceux de Detling et al (1986) sur Agropyron smithii pour qui les plantes adaptées au pâturage sont toujours moins productives lorsqu'elles ne sont pas défoliées. Ils divergent toutefois de ceux qu'obtiennent Aarssen et Turkington (1987) sur Holcus lanatus et Lolium perenne. II ne semble donc pas possible de dégager de règle valable quelles que soient les espèces.

\section{Effet de la thiamine}

Selon de nombreuses références citées par McNaughton (1985), la thiamine interviendrait comme un promoteur de croissance racinaire et ne stimulerait donc la production des parties aériennes que de matière indirecte. La thiamine est synthétisée dans le feuillage de toutes les plantes et nous considérons, ici, le cas d'une source exogène par l'intermédiaire de la salive. D'autres auteurs considèrent que la salive pourrait agir sans mimer des phytohormones mais par son contenu en sels (Detling et al, 1981). Les résultats des quelques études consacrées à ce sujet sont variés et résumés dans le tableau III.

D'après nos résultats, la thiamine n'a pas d'effet significatif sur la repousse du dactyle. Reardon et al (1974), puis McNaughton (1985), ont montré que l'effet stimulant était plus net si la défoliation était modérée voire absente. Nous n'observons pas cette tendance qui, par ailleurs, laisse perplexe quant à l'interprétation évolutive : dans la nature, la salive n'est-elle pas déposée essentiellement sur les plantes défoliées ? Nous ne notons pas, non plus, de différence selon l'expérience du pâturage qu'ont connue les populations. Sur ce dernier point, rappelons que l'étude la plus fine actuellement menée ne porte que sur une comparaison de 2 espèces, il est vrai phylogénétiquement proches Sporobolus ioclados (commune dans les sites très pâturés) et $S$ pyramidalis (McNaughton, 1985); aucune étude, à notre connaissance ne portait sur la réaction du dactyle. D'un point de vue pratique, il semble bien que l'on puisse écarter un effet de la salive sur le dactyle lorsque l'on tente de mimer le pâturage par une défoliation mécanique. Nos résultats sont donc concordants avec ceux de Detling et al (1980) et nous sommes d'accord avec leur principale conclusion, à savoir que l'importance quantitative de l'effet du dépôt de salive est mineure.

La tendance est tout de même à considérer puisque proche du seuil de signification : l'effet en serait négatif (signe observé à chaque fois que l'écart au témoin est très net), soit l'opposé de la majorité des résultats rapportés dans la littérature (une exception : Rhoades, 1985).

\section{Effet de l'histoire du pâturage (population d'origine)}

L'effet principal du facteur «population» ainsi que l'interaction "population $x$ coupe" sont donc significatifs en terme statistique. Toutefois, étant donné la petite taille de notre échantillon de génotypes, et le très grand nombre d'individus génétiques présents au sein de chaque parcelle, il nous paraît hasardeux d'affirmer que biologiquement les populations diffèrent; ce point devrait faire l'objet d'une étude complémentaire. 
Nous retenons que la population ayant expérimenté le pâturage FP30 présente une forte tendance à une meilleure résistance à la coupe par rapport à celle qui n'a jamais été très pâturée.

Les comparaisons avec les données bibliographiques sont difficiles car un facteur évolutif tel que le pâturage peut prendre de nombreuses formes (combinaisons entre l'intensité, le rythme, etc.). De plus, les différences liées à la biologie des espèces étudiées limitent aussi les possibilités de comparaison. Nous rapporterons simplement 3 travaux. Le premier va dans le sens de nos résultats : Jaramillo et Detling (1988), utilisant également un plan factoriel à 3 facteurs : "population" (pâturée ou non par les chiens de prairie), «défoliation" et "compétition» montrent que les populations ne réagissent pas différemment à la défoliation, quelles que soient leurs expériences de pâturage. À l'opposé, McNeilly (1981) a pu montrer, mais à l'aide d'un protocole plus lourd, que le rendement de Poa annua habituellement pâturé, est moins réduit que celui de plantes jamais pâturées (il appliquait une coupe particulièrement sévère : durant 13 semaines, toutes les 2 semaines à $1,5 \mathrm{~cm}$ du sol). Enfin, Hayward et al (1982) obtiennent eux aussi une interaction entre "population" et "coupe" sur la base du rendement; leur expérience est également beaucoup plus lourde que la nôtre (15 coupes sur 45 populations de Lolium multiflorum).

\section{Photosynthèse lors d'une repousse}

Il est de plus en pius largement admis que la repousse dépend essentiellement de la photosynthèse et non pas de réserves carbonées qui ne joueraient un rôle déterminant que pendant les tout premiers jours suivant une défoliation très sévère (Davidson et Milthorpe, 1966; à propos du dactyle; Richards et Caldwell, 1985, à propos d'Agropyron et pour de nombreuses références sur le sujet). Par le choix de cette variable "cinétique de la photosynthèse", nous testons donc une première hypothèse pour expliquer d'éventuelles différences dans les biomasses cumulées. La variété des cinétiques observées ne permet pas de conclure quant à d'éventuelles différences de vitesses de reprise de l'activité photosynthétique.

L'ordre de grandeur de l'activité photosynthétique que nous mesurons est cohérent avec les données obtenues par d'autres auteurs sur le dactyle (Eagles et Otham, 1978a, 1978b; Etherington, 1984; Roy, in Saugier, 1986).

II se confirme que le niveau d'activité photosynthétique est fortement déterminé génétiquement. La similitude des mesures (fig 4) correspondant à un même génotype, qu'il ait reçu ou non de la thiamine, en témoigne mieux encore que les cinétiques de $P n$.

Eagles et Otham (1978a) avaient noté également des différences significatives sur ce caractère entre des populations de dactyle différenciées sous des latitudes différentes.

\section{Le mécanisme de la repousse : liens entre photosynthèse et biomasse}

II ressort de la confrontation des résultats de biomasse avec ceux d'activité photosynthétique une observation a priori paradoxale : le génotype $D$, qui recupère le moins bien en terme de biomasse, recouvre pourtant une forte activité photosynthétique lors de la $3^{e}$ repousse. Nous avons tout d'abord vérifié que cette originalité ne provenait pas d'un simple port particulièrement prostré de ce génotype. La coupe étant faite à une hauteur constante par rapport au sol, une plante rampante connaîtrait une défoliation moins sévère qu'une plante érigée; la comparaison des biomasses résiduelles à l'issue de la dernière coupe (tableau I) permet d'écarter cette hypothèse, puisque le clone $D$ se situe dans la moyenne des autres.

Par contre, l'examen du nombre de talles fertiles coupées à chaque opération explique de manière satisfaisante la réaction singulière du génotype $D$. Celui-ci a été amputé de 7 (avec thiamine) et 6 talles fertiles (sans thiamine) alors que tous les autres génotypes n'ont été amputés que de 0-2 talles fertiles: les plants témoins non coupés (base 100 de la fig 1) ont pu accumuler de la biomasse dans les tiges et les graines alors que les plantes coupées ont été très sérieusement déprimées durant les premières semaines. Par contre, durant les 15 derniers j de l'expérience, ce génotype produisant plus de tissus jeunes que les autres (environ $20 \%$ de la biomasse totale contre $10 \%$ ). Or les tissus jeunes sont ceux qui présentent le bilan photosynthèse - respiration le plus élevé (McNaughton, 1986) et la forte activité photosynthétique par unité de poids sec s'expliquerait ainsi. 
Le cas particulier du clone $D$ conduit à souligner l'importance de la structure morphologique correspondant au stade phénologique des plantes dans l'examen de leurs réponses à la défoliation. Plus généralement, les différences entre génotypes que nous avons mises en évidence sont peut-être liées essentiellement à des différences intergénotypiques de phénologie. Le phénomène de surcompensation est donc fondamentalement dépendant de la date à laquelle est effectué le bilan de MS produite et il ne nous paraît pas opportun de considérer ce phénomène comme un caractère adaptatif particulier. Les faibles différences de $P n$ (exprimée par unité de biomasse) entre les 3 autres génotypes suggèrent d'ailleurs une variabilité réduite des réactions physiologiques spécifiques à la défoliation. Cette position est également celle de McNaughton (1974), Detling et Painter (1983), Olson et Richards (1988a, 1988b, 1988c), mais mériterait des travaux destinés à évaluer l'importance relative des mécanismes mis en jeux. D'ores et déjà, on peut émettre l'hypothèse que la sélection naturelle pour des types adaptés au pâturage opère plus particulièrement sur le nombre de talles, la date de leurs différenciations en talles fertiles ou encore la vitesse de renouvellement des feuilles (Hunt, 1970). Une étude morphométrique sur du matériel récolté dans les mêmes stations et transplanté à Toulouse a été effectuée dans le but de tester certains de ces points.

\section{REMERCIEMENTS}

Ce travail de recherche a été financé par des contrats du ministère français de la Recherche ( $n^{\circ}$ 88R074) et du ministère de l'Environnement-Comité EGPN ( $n^{\circ}$ 2632A) ainsi que par l'université Paul Sabatier, Toulouse III.

Nous remercions $\mathrm{P}$ Jacquard (CEFE, Montpellier) qui a bien voulu relire une première version de cet article ainsi que A Moisan (INRA, Toulouse) pour son aide concernant l'analyse statistique.

\section{RÉFÉRENCES}

Aarssen LW, Turkington R (1987) Responses to defoliation in Holcus lanatus, Lolium perenne and Trifolium repens from three different-aged pastures. Can J Bot 65, 1364-1370

Archer S, Tieszen L (1980) Growth and physiological responses of tundra plants to defoliation. Arct Alp Res 12, 531-552
Balent G (1986) Modélisation de l'évolution des surfaces pastorales dans les Pyrénées centrales. Mise au point d'un référentiel micro-régional de diagnostic au niveau de la parcelle. Cah Rech Dév 9/10, 92-99

Balent G, Duru M (1984) Influence des modes d'exploitation sur les caractéristiques et l'évolution des surfaces pastorales : cas des Pyrénées Centrales. Agronomie 4, 113-124

Charles AH (1961) Differential survival of cultivars of Lolium, Dactylis and Phleum. J Br Grassl Soc 16, 69-75

Davidson JL, Milthorpe FL (1966) Leaf growth in Dactylis glomerata following defoliation. Ann Bot 30 , 173-184

Després $P$, Soulas C (1981) Contribution à l'étude de l'élevage pyrénéen : le pâturage hivernal. Mémoire Fin Études ENSA Montpellier INRA-SAD Toulouse, $104 \mathrm{p}$

Detling JK, Painter EL (1983) Defoliation responses of western wheatgrass populations with diverse histories of prairie dog grazing. Oecologia Berl 57, 65-71

Detling JK, Dyer MI, Winn DT (1979) Net photosynthesis, root-respiration and regrowth of Bouteloua gracilis following simulated grazing. Oecologia Berl 41, 127-134

Detling JK, Dyer MI, Procter-Gregg C, Winn DT (1980) Plant-herbivore interactions: examination of potential effects of bison saliva on regrowth of Bouteloua gracilis (HBK) Lag Oecologia Berl 45, 26-31

Detling JK, Ross CW, Walmsley MH, Hilbert DW, Bonilla CA, Dyer MI (1981) Examination of North American bison saliva for potential plant growth regulators. J Chem Ecol 7, 239-246

Detling JK, Painter EL, Coppock DL (1986) Ecotypic differenciation resulting from grazing pressure: evidence for a likely phenomenon. In: Rangelands: a resource under siege, Proc Int rangeland Congr 1318 May 1984, Adelaïde Australie (Joss PJ, Lynch PW, Williams OB, eds) Austr Acad Sci, Camberra 431-433

Dirzo R (1984) Herbivory: A phytocentric overview. In: Perspective on Plant Population Ecology (Dirzo R, Sarukhan J, eds) Sinauer Assoc Publ, Sunderland, 141-165

Donald CM (1963) Competition among crops and pasture plants. Adv Agron 15, 1-118

Dunn JE (1981) Data-based transformations in Multivariate analysis. In: The Use of Multivariate Statistics in Studies of Wildlife Habitat (Capen DE, ed) USDA, For Serv Gen Tech Rep RM-87, 94-103

Eagles C, Othman OB (1987a) Physiological studies of a hybrid between populations of Dactylis glomerata from contrasting climatic regions, I- Interpopulation differences. Ann Appl Biol 89, 77-79

Eagles C, Othman OB (1978b) Physiological studies of a hybrid between populations of Dactylis glomerata from contrasting climatic regions, II- Intrapopulation variations. Ann App/ Bio/ 89, 81-88 
Etherington JR (1984) Relationships between morphological adaptation to grazing, carbon balance and waterlogging tolerance in clones of Dactylis glomerata L. New Phytol 98, 647-658

Fily M (1990) Sucession post-culturale et pression de pâturage dans les Pyrénées centrales : étude à l'échelle de l'écosystème, de la communauté et d'une population de Dactylis glomerata L prise comme modèle. Thèse de Doctorat de I'Univ Paul Sabatier, Toulouse III, $188 \mathrm{p}$

Hayward MC, Delacey IH, Tyler BF, Drake DW (1982) The application of pattern analysis for the recognition of adaptation in a collection of Lolium multiflorum populations. Euphytica 31, 383-396

Hunt WF (1970) The influence of leaf death on the rate of accumulation of green herbage during pasture regrowth. J App/ Ecol 7, 41-50

Izard M (1985) Le climat. In : Végétation des Pyrénées, notice détaillée des feuilles $69,70,71,72,76$, 77, 78 (Dupias G ed), CNRS, Paris, 17-38

Jaramillo VJ, Detling JK (1988) Grazing history, defoliation and competition: effects on shortgrass production and nitrogen accumulation. Ecology 69, 1599-1608

Kemp WB (1937) Natural selection within plant species as exemplified in a permanent pasture. $J$ Hered $28,329-333$

Madembo C (1986) Étude de la variabilité intraclonale, interclonale et intergénotypique de la croissance pondérale du dactyle (Dactylis glomerata $\mathrm{L}$ ). Thèse de I'INPL-Nancy, $140 \mathrm{p}$

McNaughton SJ (1974) Developmental control of net productivity in Typha latifolia ecotypes. Ecology 55, 864-869

McNaughton SJ (1979) Grassland-herbivore dynamics. In Serengeti: Dynamics of an ecosystem (Sinclair ARE, Norton-Griffith $M$, eds) Univ Chicago Press, Chicago, 46-81

McNaughton SJ (1983) Compensatory regrowth as a response to herbivory. Oikos 40, 329-336

McNaughton SJ (1985) Interactive regulation of grass yield and chemical properties by defoliation, a salivary chemical and inorganic nutrition. Oecologia 65, 478-486

McNaughton SJ (1986) On plants and herbivores. $A m$ Nat $129,407-416$

McNeilly $T$ (1981) Ecotypic differentiation in Poa annua: interpopulation differences in response to competition and cutting. New Phytol 88, 539-547

Nègre R (1972) La végétation du bassin de l'One (Pyrénées centrales), $5^{\circ}$ note : Les reposoirs, les groupements hygrophiles et les prairies de fauche. Bol Soc Broteriana, Vol XLVI, 271-343
Olson BE, Richards JH (1988a) Annual replacement of the tillers of Agropyrum desertorum following grazing. Oecologia 76, 1-6

Olson BE, Richards JH (1988b) Spatial arrangement of tiller replacement in Agropyron desertorum following grazing. Oecologia 76, 7-10

Olson BE, Richards JH (1988c) Tussock regrowth after grazing: intercalary meristem and axillary bud activity of tillers of Agropyron desertorum. Oikos 51, 374-382

Peterson RA (1962) Factors affecting resistance to heavy grazing in needle-and-thread grass. $J$ Range Manage 15, 183-189

Reardon PO, Leinweber CL, Merill LB (1972) The effect of bovine saliva on grasses. J Anim Sci 34, 897-898

Reardon PO, Leinweber CL, Merill LB (1974) Response of sideoats gramma to animal saliva and thiamine. J Range Manage 27, 400-401

Rebischung J (1953) Étude de la variabilité des populations naturelles françaises de Dactyle. Ann Amélior Plant 3, 311-349

Rhoades DF (1985) Offensive-defensive interactions between herbivores and plants: Their relevance in herbivore population dynamics and ecological theory. Am Nat 125, 205-238

Richards JH, Caldwell MM (1985) Soluble carbohydrates, concurrent photosynthesis and efficiency in regrowth following defoliation: a field study with Agropyron species. J App/ Ecol 22, 907-920

Rognli OA (1987) Genetic variation in artic populations of timothy (Phleum pratense L) II. Phenotypic stability of seed production. Hereditas 107, 75-94

Saugier B (1986) Variabilité de la réponse photosynthétique aux facteurs du milieu. Bull Soc Écophysiol 11, 1-22

Sestak Z, Catsky J, Jarvis PG (1971) Plant photosynthetic production, Manual of methods. Dr W Junk NV, La Haye

Stapledon RG (1928) Cocksfoot grass (Dactylis glomerata $L$ ) ecotypes in relation to the biotic factor. Ecology 16, 71-104

Tinker MAH (1930) The effect of cutting to ground level upon the growth of established plants of Dactylis glomerata and Phleum pratense. Welsh $J$ Agric 6, 182-198

Van Dijk GE (1955) The influence of sward age and management on the type of timothy and cocksfoot. Euphytica 4, 83-93

Williams GJ III, Kemp PR (1978) Simultaneous measurement of leaf and root gas exchange of short grass prairie species. Bot Gaz 139, 150-157 